\title{
Molecules and functions of aesculus chinensis bunge bark volatiles
}

\author{
Yi-YangLi' ${ }^{1}$, Xiangmeng Chen², Muhammad Aqeel Ashraf ${ }^{3}$, Zhenling Liu ${ }^{4}$, Huitao Bi ${ }^{1}$, Dongfang Zheng ${ }^{1}$, \\ Yong Zhao', Wan-Xi Peng'* \\ ${ }^{1}$ School of Forestry, Henan Agricultural University, Zhengzhou 450002, China, ${ }^{2}$ School of Chemical Engineering and Energy, Zhengzhou \\ University, Zhengzhou 450001, China, ${ }^{3}$ Department of Geology Faculty of Science, University of Malaya, 50603 Kuala Lumpur, Malaysia, \\ ${ }^{4}$ School of Management, Henan University of Technology, Zhengzhou 450001, China
}

\section{A B S T R A C T}

\begin{abstract}
This paper analyzes the organic solvent extracts of Aesculus chinensis Bunge Bark using TG and Py-GC-MS. The pyrolysis products were analyzed by GC-MS. The results showed that the extracts of Aesculus chinensis Bunge Bark contain a large number of biologically active components, including hydrocarbons, acids, ethers, alcohols, and phenols. The main components in the extract of Aesculus chinensis Bunge Bark are healthy and abundant; the main representatives of the active ingredients are Scopoletin Vanillin, Eugeno, Pyridine, p-Cresol, Indoles, Maltol and Furfural. There are potential applications in bioenergy, biopharmaceuticals, cosmetics, skin care products, and spices.
\end{abstract}

Keywords: Aesculus chinensis Bunge Bark; TG; Py-GC-MS

\section{INTRODUCTION}

Natural wood is a low cost and abundant material that has been used as a structural material for construction and furniture construction for thousands of years. Partial removal of lignin and hemicellulose from natural wood, followed by hot pressing, causes the cell wall to collapse completely and Naturally fully densified wood with highly aligned cellulose nanofibers (Song, et al., 2018; Fratzl, 2018). With age, the time when trees can sustain timber production remains an open question (Sillett, et al., 2015; Tollefson, 2017; Wu, et al., 2016; Ranaivoson, et al., 2017), Biomass is a renewable energy source that can be used as a substitute for fossil fuels for the production of heat energy. Wood biomass is currently the most energy-efficient energy source (Janevski, 2016; Organization, 2002). Using woody biomass as an energy source for social, economic and environmental aspects, it has been found that burning wood particles is beneficial (Nishiguchi and Tabata, 2016; Gołos and Kaliszewski, 2015), thermochemical conversion of biomass is a promising technology because biomass is an environmentally friendly fuel (Eseyin et al., 2015), Wood's lignocellulosic biomass is abundant, natural and renewable materials, and forest residues are the changes in bark ash content of post-harvest and post-harvested wood (including tree tops and bark) compared to wood parts (Abdul Halim and Yong, 2017; Haruna and Hanafiah, 2018). Great. Wood usually has a relatively low ash content, while bark has a rather high ash content (Abe, Yamada and Ohno, 2014; Álvarez, Reyes-Sosa and Díez, 2016; Nosek, Holubcik and Jandacka, 2015). Forest biomass is one of the renewable energy sources used to generate electricity, the feasibility and cost of using forest biomass to generate electricity depend on the long-term availability, cost and quality of biomass, improve the efficiency of power plants, manage forest management, and reduce transport distances (Atikuzzamman et al., 2018; Abdur Razzak et al., 2018; Fahim and Sathi, 2018). May reduce the respiratory effects of bioenergy systems (Shabani and Sowlati, 2013; Weldu, Assefa and Jolliet, 2017). The dramatic increase in U.S. wood energy consumption (16 times growth in 2060) and the high global GDP growth scenario will transform U.S. forest land into a large carbon source by 2050. This can be achieved by increasing the use of wood to promote energy use. (Nepal, et al., 2012; Roy and Corscadden, 2012). Charcoal biomass charcoal is used as an alternative fuel in direct carbon fuel cells. Wood biomass is carbon-free and recycled. In the future, wood biomass enhancement can

\footnotetext{
*Corresponding author:

Wan-Xi Peng, School of Forestry, Henan Agricultural University, Zhengzhou 450002, China. E-mail: pengwanxi@163.com
}

Revised: 21 December 2017; $\quad$ Accepted: 01 May 2018 
also be used as a solvent (Ahn, et al., 2013; Wu et al., 2015; Muhammad, Man and Mohamad Azmi, 2012).

With the strengthening of people's awareness of environmental protection, the harm of organic volatiles VOCs to the environment has received increasing attention (Chen, 2003), The generation and release of volatile degradation products during mild pyrolysis of wood is important for better understanding of the thermal degradation mechanism (Candelier, et al., 2013; Bryś, et al., 2016). There are many beneficial volatiles in wood. For example, the volatiles in Chinese fir contain many beneficial components, especially various terpenoids. Most of them have good physiological activity and aromatherapy, such as antibacterial, anti-inflammatory, and antimony (Devora Isiordia et al, 2017; Fu and Liu, 2017; Guerrini et al, 2018; Kim et al, 2018; Marvi et al, 2016; Yang et al, 2017). Antitussive, and relieve psychological tension and fatigue, it feels natural, relaxed, comfortable, happy, etc(Sun, Peng and Zhang, 2004); Some may also release a large amount of methanol, formic acid, acetic acid, aldehydes and terpenes during the drying process of Pinus massoniana. The concentration of VOCs emitted from the exhaust of Pinus massoniana during drying is relatively large and has a certain degree of environmental impact. Impact (Lan, 2007); Some organic volatiles of trees will attract some pests and cause tree death (Martini, et al., 2015). In this paper, we study the use of FT-IR, GC-MS, TG and Py-GC-MS techniques to study the organic volatiles of Aesculus chinensis Bunge Bark for research and explore its potential as a sustainable energy source.

\section{MATERIAL AND METHODS}

\section{Experimental materials}

Samples were collected from the Xixia Forest District in Henan Province. The samples were processed into powders, and four extracts were respectively extracted with ethanol, methanol, benzene/ethanol (1:1) and ethanol/ methanol (1:1), which were named C1, C2, C3, and C4 samples, respectively.

\section{Experimental methods}

TG Analysis: The samples of Aesculus chinensis Bunge Bark were analyzed by thermogravimetric analyzer (TGA Q50 V20.8 Build 34). The nitrogen release rate was $60 \mathrm{ml} / \mathrm{min}$. The temperature program of $\mathrm{TG}$ started at $30^{\circ} \mathrm{C}$ and rose to $300^{\circ} \mathrm{C}$ at a rate of $5^{\circ} \mathrm{C} / \mathrm{min}$ (Ge et al., 2017; Peng, et al., 2017; Jiang et al., 2017).

Py-GC-MS Analysis: Catalyzed and pretreated samples were analyzed by pyrolysis-gas chromatography-mass spectrometry (CDS5000-Agilent 7890B-5977A ISQ). Carrier gas for high purity helium, pyrolysis temperature of $500^{\circ} \mathrm{C}$, heating rate of $20^{\circ} \mathrm{C} / \mathrm{ms}$, pyrolysis time of 15 s. Pyrolysis product transfer line and injection valve temperature was set at $300^{\circ} \mathrm{C}$; HP-5MS column; capillary column $(60 \mathrm{~m} \times 250 \mu \mathrm{m} \times 0.25 \mu \mathrm{m})$; parallel mode, the split ratio 1:60, shunt speed $50 \mathrm{~mL} / \mathrm{min}$. The temperature of the GC program was raised from $40^{\circ} \mathrm{C}$ for 2 minutes, raised to $120^{\circ} \mathrm{C}$ at a rate of $5^{\circ} \mathrm{C} /$ minute and then raised to $200^{\circ} \mathrm{C}$ at a rate of $10^{\circ} \mathrm{C} /$ minute for 15 minutes. Ion source (EI) temperature of $230^{\circ} \mathrm{C}$, the scanning range of 28 amu-500amu (Xu et al., 2018; Wang et al., 2018; Liu et al., 2018; Ge et al., 2018; Zhou et al., 2016).

\section{RESULTS AND DISCUSSION}

\section{Analysis of TGA and DTG}

Fig 1 shows the TGA and DTG curves of Aesculus chinensis Bunge Bark. The thermogravimetry measures the quality change and the rate of change of the schizocarps, and at the same time determines the broad application prospects of its resources (Peng et al., 2017; Li et al., 2017; Min et al., 2018; Jiang et al., 2018). Therefore, thermal stability analysis is also an effective method to evaluate the application of Aesculus chinensis Bunge Bark resources. In order to study Aesculus chinensis Bunge Bark, we conducted a TGA test, as shown in Fig. 1, TGA curve and DTG curve (Jiang, Ge and Peng, 2017; Ge

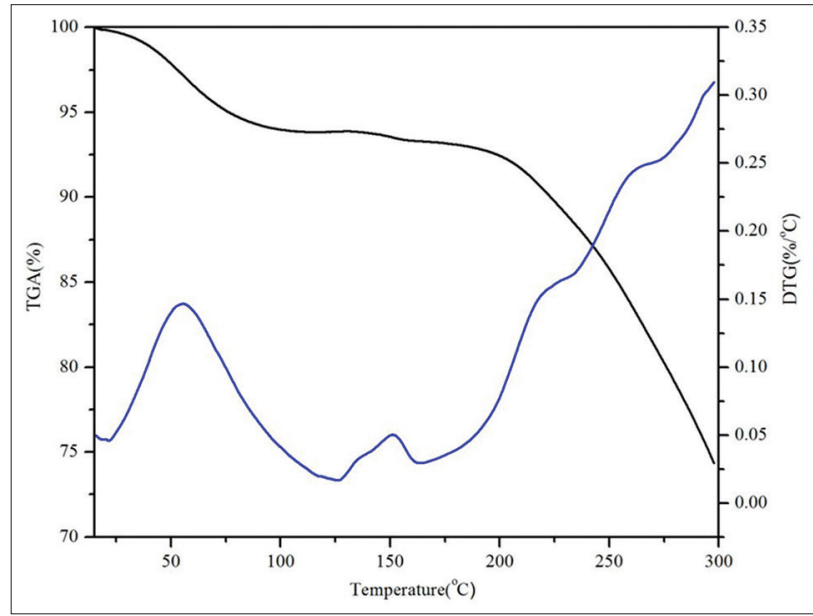

Fig 1. TGA and DTG thermal curves of Aesculus chinensis.

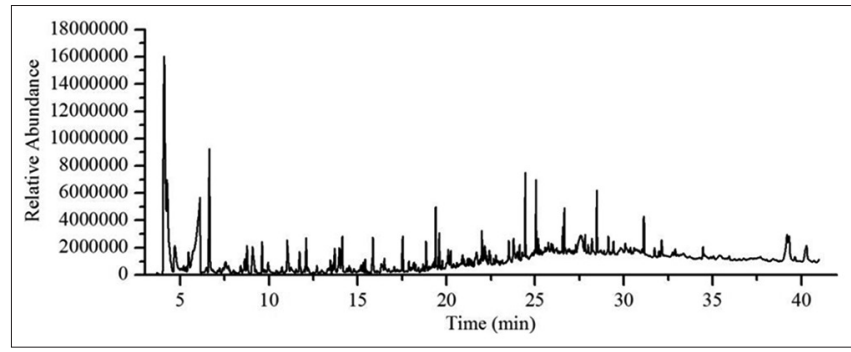

Fig 2. Total ion chromatograms of Aesculus chinensis Bunge Bark by Py-GC-MS. 
Table 1: Py-GC-MS analysis of aesculus chinensis bunge bark

\begin{tabular}{|c|c|c|c|}
\hline No. & Retention time (min) & Peak area (\%) & Component \\
\hline 1 & 3.70 & 0.04 & 2-Pentanamine \\
\hline 2 & 4.09 & 8.33 & Ethyne, fluoro- \\
\hline 3 & 4.26 & 4.20 & 1,4-Dioxane-2,6-dione \\
\hline 4 & 4.70 & 1.44 & Diazene, dimethyl- \\
\hline 5 & 4.92 & 0.18 & Acetic acid, methyl ester \\
\hline 6 & 5.06 & 0.25 & 1,3-Cyclopentadiene \\
\hline 7 & 5.18 & 0.24 & Propanal, 2-methyl- \\
\hline 8 & 5.30 & 0.20 & 1-Propanol \\
\hline 9 & 5.46 & 0.69 & 2,3-Butanedione \\
\hline 10 & 5.71 & 0.88 & Acetic acid \\
\hline 11 & 5.73 & 0.17 & Acetic acid \\
\hline 12 & 6.11 & 5.53 & Acetic acid \\
\hline 13 & 6.47 & 0.32 & (Z)-1,3-Butadien-1-ol \\
\hline 14 & 6.64 & 2.61 & 2-Propanone, 1-hydroxy- \\
\hline 15 & 6.84 & 0.11 & Ethanone, 1-cyclopropyl- \\
\hline 16 & 7.10 & 0.08 & Acetamide, $\mathrm{N}$-(aminoiminomethyl)- \\
\hline 17 & 7.14 & 0.04 & 3-Butenoic acid \\
\hline 18 & 7.22 & 0.19 & 2,3-Pentanedione \\
\hline 19 & 7.41 & 0.21 & 1,2-Ethanediol \\
\hline 20 & 7.55 & 0.53 & Furan, 2,5-dimethyl- \\
\hline 21 & 7.66 & 0.07 & Propanoic acid \\
\hline 22 & 7.69 & 0.06 & Propanoic acid \\
\hline 23 & 7.71 & 0.13 & Propanoic acid \\
\hline 24 & 7.94 & 0.05 & Acetamide, N-2-propynyl- \\
\hline 25 & 8.01 & 0.07 & Propanoic acid, 2-oxo-, methyl ester \\
\hline 26 & 8.40 & 0.26 & 1H-Pyrrole, 1-methyl- \\
\hline 27 & 8.54 & 0.09 & $\mathrm{~N}, \mathrm{~N}$-Dimethylaminoethanol \\
\hline 28 & 8.61 & 0.30 & Pyridine \\
\hline 29 & 8.76 & 0.71 & Pyrrole \\
\hline 30 & 9.07 & 0.99 & 2-Propanone, 1-hydroxy- \\
\hline 31 & 9.27 & 0.13 & 2-Butenal, 2-methyl-, (E)- \\
\hline 32 & 9.61 & 0.60 & Propanoic acid, 2-oxo-, methyl ester \\
\hline 33 & 9.77 & 0.10 & Cyclopentanone \\
\hline 34 & 9.94 & 0.28 & 3-Amino-s-triazole \\
\hline 35 & 10.02 & 0.07 & 4-Methyl-3,7,9-trioxabicyclo $(4,2,1)$ nonane \\
\hline 36 & 10.39 & 0.07 & 3-Furaldehyde \\
\hline 37 & 10.46 & 0.05 & 1H-Pyrrole, 1-ethyl- \\
\hline 38 & 10.61 & 0.03 & Pyrrolidine \\
\hline 39 & 10.66 & 0.04 & Pyridine, 2-methyl- \\
\hline 40 & 10.74 & 0.15 & Pyrazine, methyl- \\
\hline 41 & 10.92 & 0.05 & 2-Vinylethyl acetate \\
\hline 42 & 11.03 & 0.56 & Furfural \\
\hline 43 & 11.08 & 0.37 & 2-Cyclopenten-1-one \\
\hline 44 & 11.23 & 0.16 & 1H-Pyrrole, 3-methyl- \\
\hline 45 & 11.30 & 0.09 & 1-Butene, 3-methyl- \\
\hline 46 & 11.53 & 0.11 & 1H-Pyrrole, 3-methyl- \\
\hline 47 & 11.72 & 0.52 & 2-Furanmethanol \\
\hline 48 & 11.99 & 0.09 & Benzene, 1,3-dimethyl- \\
\hline 49 & 12.09 & 0.57 & 2-Propanone, 1-(acetyloxy)- \\
\hline 50 & 12.20 & 0.17 & 2(3H)-Furanone, 5-methyl- \\
\hline 51 & 12.60 & 0.02 & Carbamic acid, phenyl ester \\
\hline 52 & 12.69 & 0.13 & Cyclopent-4-ene-1,3-dione \\
\hline 53 & 12.96 & 0.04 & Styrene \\
\hline 54 & 13.32 & 0.14 & 2-Butenoic acid, methyl ester, (E)- \\
\hline 55 & 13.46 & 0.30 & 2-Cyclopenten-1-one, 2-methyl- \\
\hline
\end{tabular}


Table 1: (Continued)

\begin{tabular}{|c|c|c|c|}
\hline No. & Retention time (min) & Peak area $(\%)$ & Component \\
\hline 56 & 13.59 & 0.13 & Ethanone, 1-(2-furanyl)- \\
\hline 57 & 13.71 & 0.59 & 2(5H)-Furanone \\
\hline 58 & 13.96 & 0.72 & Diisoamyl ether \\
\hline 59 & 14.13 & 0.86 & 2-Cyclopenten-1-one, 2-hydroxy- \\
\hline 60 & 14.38 & 0.09 & Pyridine, 3,5-dimethyl- \\
\hline 61 & 14.52 & 0.13 & 2(5H)-Furanone, 5-methyl- \\
\hline 62 & 14.60 & 0.07 & 2,5-Furandione, dihydro-3-methylene- \\
\hline 63 & 14.78 & 0.09 & Cycloheptanone \\
\hline 64 & 15.02 & 0.04 & Thiazole, 4,5-dihydro-2-methyl- \\
\hline 65 & 15.09 & 0.06 & 1,4-Pentanediol \\
\hline 66 & 15.17 & 0.16 & 2,3-Pentanedione \\
\hline 67 & 15.31 & 0.26 & 2-Furancarboxaldehyde, 5-methyl- \\
\hline 68 & 15.42 & 0.22 & 2-Cyclopenten-1-one, 3-methyl- \\
\hline 69 & 15.54 & 0.03 & Pyridine, 2-ethenyl- \\
\hline 70 & 15.80 & 0.11 & 1-(Dimethylamino) pyrrole \\
\hline 71 & 15.86 & 0.68 & Phenol \\
\hline 72 & 16.06 & 0.05 & Hexanoic acid \\
\hline 73 & 16.32 & 0.10 & Aziridine, 2-methyl-3-(1-methylethyl)-, trans- \\
\hline 74 & 16.35 & 0.28 & 3-Amino-1,2,4-triazole-5-carboxylic acid \\
\hline 75 & 16.50 & 0.28 & 3-Cyclobutene-1,2-dione, 3,4-dihydroxy- \\
\hline 76 & 16.58 & 0.14 & 2-Furanone, 2,5-dihydro-3,5-dimethyl \\
\hline 77 & 16.75 & 0.10 & 1H-Pyrrole, 2-ethyl-4-methyl- \\
\hline 78 & 17.06 & 0.11 & 6-Azacytosine \\
\hline 79 & 17.16 & 0.05 & Tricyclo[4.2.1.1 (2,5)]decane \\
\hline 80 & 17.22 & 0.06 & 2-Cyano-1-hexene \\
\hline 81 & 17.29 & 0.05 & 1,3-Dimethyl-1-cyclohexene \\
\hline 82 & 17.38 & 0.05 & 2-Propyl-1-pentanol, pentafluoropropionate \\
\hline 83 & 17.54 & 0.79 & 1,2-Cyclopentanedione, 3-methyl- \\
\hline 84 & 17.73 & 0.04 & 3-Heptyne, 2,2-dimethyl- \\
\hline 85 & 17.88 & 0.25 & 2-Cyclopenten-1-one, 2,3-dimethyl- \\
\hline 86 & 18.02 & 0.10 & 4-Methyl-5H-furan-2-one \\
\hline 87 & 18.16 & 0.28 & 1,3-Dioxol-2-one, 4,5-dimethyl- \\
\hline 88 & 18.25 & 0.15 & Phenol, 2-methyl- \\
\hline 89 & 18.42 & 0.09 & 2-Cyclopenten-1-one, 2-hydroxy-3,4-dimethyl- \\
\hline 90 & 18.58 & 0.06 & Ethanone, 1-(1H-pyrrol-2-yl)- \\
\hline 91 & 18.68 & 0.15 & 2,5-Dimethylfuran-3,4(2H,5H)-dione \\
\hline 92 & 18.75 & 0.05 & Acetophenone \\
\hline 93 & 18.85 & 0.58 & p-Cresol \\
\hline 94 & 19.01 & 0.10 & 2-Cyclopenten-1-one, 3-ethyl- \\
\hline 95 & 19.09 & 0.06 & Heptanoic acid \\
\hline 96 & 19.12 & 0.07 & Valeraldehyde, 2,2-dimethyl-, oxime \\
\hline 97 & 19.21 & 0.19 & Furyl hydroxymethyl ketone \\
\hline 98 & 19.28 & 0.20 & 6-Amino-1,3,5-triazine-2,4(1H,3H)-dione \\
\hline 99 & 19.39 & 0.90 & Phenol, 2-methoxy- \\
\hline 100 & 19.47 & 0.08 & Cyclohexanol, 2,2-dimethyl- \\
\hline 101 & 19.59 & 0.83 & Diazene, [1-(2,2-dimethylhydrazino) ethyl] ethyl- \\
\hline 102 & 19.77 & 0.22 & Propanoic acid, 2-chloro-, pentyl ester \\
\hline 103 & 19.99 & 0.17 & 3-Pyridinol \\
\hline 104 & 20.03 & 0.09 & 3-Pyridinol \\
\hline 105 & 20.10 & 0.56 & Maltol \\
\hline 106 & 20.25 & 0.43 & 2-Cyclopenten-1-one, 3-ethyl-2-hydroxy- \\
\hline 107 & 20.39 & 0.13 & 10-Methylundecan-4-olide \\
\hline 108 & 20.47 & 0.13 & 5-[3-Methyl-2-furyl] hydantoin \\
\hline 109 & 20.59 & 0.18 & (1,4,4-Trimethyl-cyclohex-2-enyl)-acetic acid \\
\hline 110 & 20.69 & 0.09 & 10-Methylundecan-4-olide \\
\hline
\end{tabular}


Table 1: (Continued)

\begin{tabular}{|c|c|c|c|}
\hline No. & Retention time (min) & Peak area $(\%)$ & Component \\
\hline 111 & 20.75 & 0.13 & Formaldehyde, methyl(2-propynyl) hydrazone \\
\hline 112 & 20.82 & 0.16 & 1,4-Benzodioxin, octahydro-2-methylene-, trans- \\
\hline 113 & 20.90 & 0.40 & 4H-Pyran-4-one, 2,3-dihydro-3,5-dihydroxy-6-methyl- \\
\hline 114 & 20.99 & 0.21 & $3(2 \mathrm{H})$-Furanone, dihydro-5-methyl- \\
\hline 115 & 21.06 & 0.12 & Tetracyclo[5.3.0.0<2,6>.0<3,10>]deca-4,8-diene \\
\hline 116 & 21.19 & 0.30 & 2H-Pyran-2-one, tetrahydro-3,5-dimethyl- \\
\hline 117 & 21.30 & 0.23 & Phenol, 4-ethyl- \\
\hline 118 & 21.34 & 0.12 & 5,8-Decadien-2-one, 5,9-dimethyl-, (E)- \\
\hline 119 & 21.41 & 0.17 & 2-Butenoic acid, 3-amino-, ethyl ester \\
\hline 120 & 21.50 & 0.16 & Octanoic acid \\
\hline 121 & 21.69 & 0.94 & 2-Penten-1-ol, (E)- \\
\hline 122 & 21.89 & 0.26 & Pentyl acetoacetate \\
\hline 123 & 21.95 & 0.12 & 7-Thiabicyclo[4.2.1]nonane \\
\hline 124 & 22.00 & 0.57 & Creosol \\
\hline 125 & 22.07 & 0.10 & 4,7,7-Trimethyl-5-(tetrahydropyran-2-yloxy)-bicyclo[2.2.1]heptan-2-one \\
\hline 126 & 22.15 & 0.87 & Catechol \\
\hline 127 & 22.27 & 0.32 & Catechol \\
\hline 128 & 22.42 & 0.40 & 1,4:3,6-Dianhydro-.alpha.-d-glucopyranose \\
\hline 129 & 22.47 & 0.21 & Benzofuran, 2,3-dihydro- \\
\hline 130 & 22.66 & 0.28 & Heptadecanal \\
\hline 131 & 22.80 & 0.33 & 2,3-Anhydro-d-mannosan \\
\hline 132 & 22.85 & 0.12 & 2-Isopropoxyphenol \\
\hline 133 & 22.91 & 0.15 & chrysanthemyl 2-hydroxy-3-methylbutanoate \\
\hline 134 & 23.05 & 0.13 & Carbamic acid, (4-nitrophenyl)-, phenylmethyl ester \\
\hline 135 & 23.16 & 0.26 & 2-Cyclohexen-1-one, 3,4,4-trimethyl- \\
\hline 136 & 23.26 & 0.14 & 5,6,6-Trimethyl-5-(3-oxobut-1-enyl)-1-oxaspiro[2.5]octan-4-one \\
\hline 137 & 23.29 & 0.10 & Benzene, 1,3-dimethoxy-2-nitro- \\
\hline 138 & 23.35 & 0.11 & 7-Oxabicyclo[4.1.0]heptane, 2-methylene- \\
\hline 139 & 23.42 & 0.15 & 1,2-Benzenediol, 4-methyl- \\
\hline 140 & 23.51 & 0.95 & 1,2-Benzenediol, 3-methoxy- \\
\hline 141 & 23.78 & 0.78 & Phenol, 4-ethyl-2-methoxy- \\
\hline 142 & 23.85 & 0.28 & Isopropylphosphonic acid, dimethyl ester \\
\hline 143 & 23.95 & 0.22 & 1,3,5,7-Cyclooctatetraene \\
\hline 144 & 24.00 & 0.55 & 1,2-Benzenediol, 4-methyl- \\
\hline 145 & 24.13 & 0.41 & Indole \\
\hline 146 & 24.27 & 0.58 & 4-Acetoxy-1,2,3,5,6,7,8,8a-octahydroazulene \\
\hline 147 & 24.44 & 1.48 & 2-Methoxy-4-vinylphenol \\
\hline 148 & 24.51 & 0.09 & 8-Tetradecyn-1-ol \\
\hline 149 & 24.56 & 0.41 & Globulol \\
\hline 150 & 24.72 & 0.31 & 2,4-Diaminophenol \\
\hline 151 & 24.78 & 0.25 & Benzenemethanol, .alpha.-ethynyl- \\
\hline 152 & 24.82 & 0.28 & Pyran, tetrahydro-2-(3-bromo-5,5-dimethyl-2-cyclohexenyl) oxy- \\
\hline 153 & 24.89 & 0.25 & $\begin{array}{l}\text { 1H-Isoindole-1,3(2H)-dione, 3a, } \\
\text { 4,5,7a-tetrahydro-4-hydroxy-3a-methyl-2-phenyl-, [3aR-(3a.alpha.,4.beta.,7a.alpha.)] }\end{array}$ \\
\hline 154 & 24.96 & 0.41 & 1,4-Benzenediol, 2-methyl- \\
\hline 155 & 25.05 & 1.30 & Phenol, 2,6-dimethoxy- \\
\hline 156 & 25.18 & 0.68 & Eugenol \\
\hline 157 & 25.28 & 0.31 & 3-Amino-2,6-dimethoxypyridine \\
\hline 158 & 25.33 & 0.39 & Phenol, 2-methoxy-4-propyl- \\
\hline 159 & 25.44 & 0.30 & 9-Borabicyclo[3.3.1]nonane, 9-(1-ethylbutyl)- \\
\hline 160 & 25.47 & 0.13 & 1H-Imidazole-4-methanol, 5-methyl- \\
\hline 161 & 25.50 & 0.11 & 2,4-Hexadiene, 2,5-dimethyl- \\
\hline 162 & 25.57 & 0.68 & 1-Benzoxepin-2(3H)-one, octahydro- \\
\hline 163 & 25.67 & 0.39 & 11-Bromoundecanoic acid \\
\hline 164 & 25.74 & 0.52 & Indole, 3-methyl- \\
\hline
\end{tabular}


Table 1: (Continued)

\begin{tabular}{|c|c|c|c|}
\hline No. & Retention time (min) & Peak area $(\%)$ & Component \\
\hline 165 & 25.78 & 0.32 & 4,5,6-Trimethyl-2-pyrimidone \\
\hline 166 & 25.86 & 0.32 & meta-Methoxybenzenethiol \\
\hline 167 & 25.91 & 0.44 & Vanillin \\
\hline 168 & 25.99 & 0.47 & 3-Allyl-6-methoxyphenol \\
\hline 169 & 26.11 & 0.31 & $\begin{array}{l}\text { 1S,3R,4S,5R,6S-1-Hydroxy-2,2,3,4,5,6-hexamethyl-8-oxo-7,9-dioxatricyclo[4.2.1.0 (3,5)] } \\
\text { nonae }\end{array}$ \\
\hline 170 & 26.15 & 0.24 & Tetrahydroionone \\
\hline 171 & 26.21 & 0.58 & 4-Nitro-3-picoline- $\mathrm{N}$-oxide \\
\hline 172 & 26.33 & 0.41 & Benzenamine, 3-methoxy- \\
\hline 173 & 26.44 & 0.43 & 1-Tetradecene, 14-bromo- \\
\hline 174 & 26.55 & 0.62 & 3,5-Dimethoxy-4-hydroxytoluene \\
\hline 175 & 26.64 & 0.96 & trans-Isoeugenol \\
\hline 176 & 26.72 & 0.37 & Spiro[4.5]decan-7-one, 1,8-dimethyl-8,9-epoxy-4-isopropyl- \\
\hline 177 & 26.84 & 0.48 & Bicyclo (3.3.1) nonane-2,6-dione \\
\hline 178 & 26.92 & 0.46 & Bicyclo[3.1.0]hexane-6-methanol, 2-hydroxy-1,4,4-trimethyl- \\
\hline 179 & 26.99 & 0.30 & Bicyclo[3.1.0]hexane-6-methanol, 2-hydroxy-1,4,4-trimethyl- \\
\hline 180 & 27.08 & 0.36 & Oleic Acid \\
\hline 181 & 27.19 & 0.31 & 12-Methyl-E, E-2,13-octadecadien-1-ol \\
\hline 182 & 27.29 & 0.52 & 5-Hepten-3-yn-2-ol, 6-methyl-5-(1-methylethyl)- \\
\hline 183 & 27.59 & 2.17 & .beta.-D-Glucopyranose, 1,6-anhydro- \\
\hline 184 & 27.61 & 1.20 & D-Allose \\
\hline 185 & 27.81 & 0.75 & Benzene, 1,2,3-trimethoxy-5-methyl- \\
\hline 186 & 27.99 & 0.38 & 2-Propanone, 1-(4-hydroxy-3-methoxyphenyl)- \\
\hline 187 & 28.20 & 1.53 & Dodecanoic acid, 1-methylethyl ester \\
\hline 188 & 28.48 & 1.30 & 2,3,5,6-Tetrafluoroanisole \\
\hline 189 & 28.64 & 0.46 & 2-Hydroxy-2,4,4-trimethyl-3-(3-methylbuta-1,3-dienyl) cyclohexanone \\
\hline 190 & 28.70 & 0.68 & 10-Methyldodec-2-en-4-olide \\
\hline 191 & 28.88 & 0.32 & Benzofuran-4(5H)-one, 6,7-dihydro-, oxime \\
\hline 192 & 29.12 & 1.08 & Phenol, 2,6-dimethoxy-4-(2-propenyl)- \\
\hline 193 & 29.25 & 0.27 & Pyrazole-5-carboxylic acid, 1-ethyl-4-nitro- \\
\hline 194 & 29.41 & 0.96 & Benzeneethanamine, 2-fluoro-4,5-dimethoxy-.beta.-hydroxy-N-isopropyl- \\
\hline 195 & 29.82 & 1.70 & 1-Chloroeicosane \\
\hline 196 & 30.08 & 1.30 & 5,7-Dimethyl-1,3-diazaadamantan-6-one Hydrazone \\
\hline 197 & 30.37 & 0.60 & 2-Pentadecanone, 6,10,14-trimethyl- \\
\hline 198 & 30.59 & 0.55 & 4-((1E)-3-Hydroxy-1-propenyl)-2-methoxyphenol \\
\hline 199 & 30.70 & 0.29 & 2,4,5-Tri-O-acetyl-3,6-di-O-methyl-D-mannonitrile \\
\hline 200 & 30.75 & 0.39 & Octyltrichlorosilane \\
\hline 201 & 30.85 & 0.51 & 2-Trimethylsilyl-1,3-dithiane \\
\hline 202 & 31.02 & 0.43 & 1,3-Benzenediol, 2,5-dimethyl- \\
\hline 203 & 31.12 & 0.98 & (E)-2,6-Dimethoxy-4-(prop-1-en-1-yl) phenol \\
\hline 204 & 31.72 & 0.63 & D-Norleucine, N-isobutoxycarbonyl-, ethyl ester \\
\hline 205 & 31.97 & 0.35 & Ethanone, 1-(4-hydroxy-3,5-dimethoxyphenyl)- \\
\hline 206 & 32.12 & 0.70 & 4-((1E)-3-Hydroxy-1-propenyl)-2-methoxyphenol \\
\hline 207 & 32.25 & 0.19 & 2(3H)-Naphthalenone, 4,4a, 5,6,7,8-hexahydro-1-methoxy- \\
\hline 208 & 32.76 & 0.45 & Tetrazolo[1,5-a] pyrimidine, 6-(3-hydroxy-2-oxopropyl)- \\
\hline 209 & 32.88 & 0.37 & 2-Pentanone, 1-(2,4,6-trihydroxyphenyl) \\
\hline 210 & 33.03 & 0.29 & Cyclotetradecane \\
\hline 211 & 33.20 & 0.28 & Hexadecenoic acid, Z-11- \\
\hline 212 & 33.35 & 0.56 & Oleic Acid \\
\hline 213 & 34.46 & 0.31 & Neophytadiene \\
\hline 214 & 34.56 & 0.24 & cis-7, cis-11-Hexadecadien-1-yl acetate \\
\hline 215 & 34.90 & 0.10 & 2-Cyclohexen-1-one, 3-(3-hydroxybutyl)-2,4,4-trimethyl- \\
\hline 216 & 35.05 & 0.09 & Pentadecanoic acid \\
\hline 217 & 35.39 & 0.26 & Docosanoic acid \\
\hline 218 & 35.93 & 0.08 & E-8-Methyl-9-tetradecen-1-ol acetate \\
\hline
\end{tabular}


Table 1: (Continued)

\begin{tabular}{lccl}
\hline No. & Retention time $(\mathbf{m i n})$ & Peak area $(\%)$ & Component \\
\hline 219 & 36.75 & 0.06 & p-Phenylenediamine, N-benzylidene-N'-phenyl- \\
220 & 37.09 & 0.02 & Pyrimidine-5-carbonitrile, 3,4-dihydro-6-(dimethylaminophenyl)-2-mercapto-4-oxo- \\
221 & 37.17 & 0.03 & 19-Norandrost-4-en-3,17-dione (8.beta.,9.beta., 10.alpha.) \\
222 & 37.70 & 0.08 & p-Phenylenediamine, N-benzylidene-N'-phenyl- \\
223 & 38.41 & 0.06 & cis-1-Chloro-9-octadecene \\
224 & 39.20 & 1.19 & n-Hexadecanoic acid \\
225 & 39.31 & 1.00 & Scopoletin \\
226 & 39.64 & 0.14 & Dibutyl phthalate \\
227 & 40.28 & 0.87 & Scopoletin \\
\hline
\end{tabular}

et al., 2018; Jiang, Ge and Peng, 2017). From the figure, we can see that the weight loss process of pyrolysis can be divided into three stages. The first stage is between 16$98^{\circ} \mathrm{C}$, which is the stage of water evaporation (Peng et al., 2016; Xie et al., 2018; Lourençon et al., 2014). The weight loss of the sample is small, and the weight loss is mainly caused by the loss of biomass. Water-induced. The second stage is between 98 and $198^{\circ} \mathrm{C}$. It is a preheating solution transition stage (Ge, et al., 2017; Peng, et al., 2017; Ge et al., 2018). The differential curve at this stage is relatively flat, indicating that the pyrolysis rate is relatively stable, and the sample begins to show obvious weightlessness. The weight loss is mainly due to the inside of the sample. A small amount of polymer depolymerization and reorganization occurred (Ge et al., 2017; Fu et al., 2009; $\mathrm{Li}$ et al., 2017). The third stage is between $198-300^{\circ} \mathrm{C}$. During the combustion of the remaining components, the cellulose and hemicellulose in Aesculus chinensis Bunge rapidly cleave and generate a large amount of volatile gases and cause weightlessness as the temperature rises (Li et al., 2017; Peng et al., 2018). These three stages show different properties with different kinetic parameters and reaction mechanisms. The final residual mass is $74.34 \%$ (Ouyang et al., 2016; Peng et al., 2016), between 16-280 ${ }^{\circ} \mathrm{C}$. Aesculus chinensis Bunge Bark has a thermogravimetric weight of only about $15 \%$. It has less thermal weight loss, a smaller mass change, and a lower rate. The TG test showed that Aesculus chinensis Bunge Bark has good processing performance and great potential for resource utilization (Chen, et al., 2017; Ge, et al., 2017).

\section{Analysis of Py-GC-MS}

According to the Py-GC-MS analysis results (Table 1), a total of 227 compounds were detected, of which the highest levels were: Ethyne, fluoro- (8.33\%). 1,4-Dioxane2,6-dione (4.20\%). Diazene, dimethyl-(1.44\%). Acetic acid (6.58\%). 2-Propanone, 1-hydroxy-(2.61\%). Pyrrole (0.71\%). 2-Propanone, 1-hydroxy-(0.99\%). Diisoamyl ether $(0.72 \%)$. 2-Cyclopenten-1-one, 2-hydroxy- $(0.86 \%)$. 1,2-Cyclopentanedione, 3-methyl-(0.79\%). Phenol, 2-methoxy-(0.90\%). Diazene, [1-(2,2-dimethylhydrazino) ethyl] ethyl - $(0.83 \%)$. Catechol $(0.87 \%)$. 1,2-Benzenediol, 3-methoxy-(0.95\%). Phenol, 4-ethyl-2-methoxy-(0.78\%). 2 -Methoxy-4-vinylphenol(1.48\%). Phenol, 2,6-dimethoxy-(1.30\%). beta.-D-Glucopyranose, 1,6-anhydro-(2.17\%). Benzene, 1,2,3-trimethoxy5-methyl-(0.75\%). Dodecanoic acid, 1-methylethyl ester(1.53\%). 2,3,5,6-Tetrafluoroanisole(1.30\%). Phenol, 2,6-dimethoxy-4-(2-propenyl)-(1.08\%). Benzeneethanamine, 2-fluoro-4,5-dimethoxy-.beta.-hydroxy-N-isopropyl-(0.96\%). 1-Chloroeicosane(1.70\%).5,7-Dimethyl-1,3-diazaadamantan6-one Hydrazone(1.30\%). (E)-2,6-Dimethoxy-4-(prop1 -en-1-yl)phenol(0.98\%). n-Hexadecanoic acid $(1.19 \%)$. Scopoletin $(1.00 \%)$.

\section{Analysis of function}

The Aesculus chinensis Bunge Bark products have certain human health functions. The analysis of Aesculus chinensis Bunge Bark using GC-MS and Py-GC-MS techniques has yielded related compounds, and by consulting relevant literature and reports, we have obtained the proven active ingredients of human health. Scopoletin regulates blood pressure and has antibacterial activity (Gnonlonfin, Sanni and Brimer, 2012). It can be used to treat bronchial diseases and caudal horns, regulate hormone balance, and help relieve anxiety and depression. In addition, studies have reported that Moxa Coccinea has analgesic, anti-inflammatory and antioxidant effects (Jamuna et al., 2015), blood pressure lowering and antispasmodic effects, especially antitumor (Liu et al., 2012; Tabana et. al., 2016; Seo et al., 2016; Zhao et al., 2012) and its activity in the prevention and treatment of hyperuricemia (Yao et al., 2012), has currently attracted widespread attention at home and abroad. It can prevent alcohol-induced Hepatic lipid accumulation (Lee et al., 2014), insecticidal activity, acaricidal activity, antibacterial activity, growth regulation, and induction of plant resistance (Hino, Okazaki and Miura, 2014).

Vanillin is a food flavoring agent, has the fragrant smell of vanilla beans and milky desire. It is an indispensable important raw material in the food additive industry. Foreign vanillin has a wide range of applications and is widely 
used in the production of pharmaceutical intermediates. Used for plant growth promoters, bactericides, lubricant defoamers, electroplating brighteners, and conductive agents for printed circuit boards (Fache, Boutevin and Caillol, 2016; Kaur and Chakraborty, 2013). Eugeno can be used as a modifier and fixatives, use colored soaps to add fragrance, use for antibacterial and lower blood pressure (Kong et al., 2013). Eugeno is a potent anesthetic (Roubach et al., 2015). Eugeno can also inhibit the growth of melanoma (Ghosh et al., 2015). Among the many derivatives of Pyridine, some are important drugs, and some are vitamins or enzymes. An important part of. The pyridine derivative isoniazid is an oral anti-tuberculosis drug, bactericide, fungicide, and organic synthesis (Moraski et al., 2015; Ayieko et al., 2014).

p-Cresol is used in organic synthesis and p-Cresol sulfate and indoxyl sulfate play an important role in renal injury (Sun, Hsu and Wu, 2013). Intestinal absorption of p-cresol and independence from renal function in patients with chronic kidney disease Is related to cardiovascular disease. Intestinal metabolism and p-cresol uptake may be therapeutic targets for reducing the risk of cardiovascular disease in patients with renal insufficiency (Poesen et al., 2014). Indoles are naturally occurring and synthetic, with a wide range of biological activities, unusual and complex molecular structures occur in their natural derivatives (Inman \& Moody, 2012). Indole naphthyridinones is an inhibitor of the bacterial Enoyl-ACP reductase FabI and FabK (Seefeld et al., 2015). Indole-3-acetic is an important plant hormone that has the ability to control plant development in a beneficial and harmful manner (Zhao, 2012; Duca et al., 2014). Maltol pretreatment effectively reduced alcoholinduced oxidative damage in a dose-dependent manner. Maltol seems to have promising antioxidant and antiinflammatory abilities (Han et al., 2015). Maltol stimulated kainic acid the neuroprotective effects of oxidative damage in the brain of mice (Kim et al., 2013), Maltol has potential as a novel neuroprotective agent for oxidative stress-related eye diseases (including glaucoma) (Hong et al., 2014). Furfural provides a promising and abundant platform for lignocellulosic biofuels (Lange et al., 2012).

\section{CONCLUSIONS}

From the above test results, it can be concluded that The TGA is divided into two stages: the first stage is the evaporation of water at low temperature, the second stage is caused by a small amount of depolymerization and reorganization of the polymer, and the third stage is the stage of coke passing through the aerobic combustion in the high temperature stage. Below $300^{\circ} \mathrm{C}$, only a small amount of hemicellulose, cellulose and lignin are pyrolyzed.
In the Py-GC-MS test, 227 compounds were identified in Aesculus chinensis Bunge Bark. From the above studies, it can be seen that the active ingredients of Aesculus chinensis Bunge Bark have antibacterial, antitumor and insecticidal activities and can be used as lignocellulosic biofuels, etc. Therefore, Aesculus chinensis Bunge Bark has better high-grade resource utilization, and the sustainable development of material resources has broad prospects.

\section{ACKNOWLEDGMENTS}

The Hunan Science Fund for Distinguished Young Scholars (16JJ1028), The authors acknowledge financial support by the new century excellent talent support program (NCET-12-0725), the Major scientific and technological achievements transformation projects of strategic emerging industries in Hunan Province (2016GK4045), and the Academician reserve personnel training plan of lift engineering technical personnel of Hunan Science and Technology Association (2017TJ-Y10).

\section{REFERENCES}

Abdul, H. K and E. L. Yong. 2018. Integrating two-stage up-flow anaerobic sludge blanket with a single-stage aerobic packedbed reactor for raw palm oil mill effluent treatment. Malays. J. Sustain. Agric. 1(1): 15-18.

Abdur, R. M., M. A. Islam, M. H. Rahman, M. A. Sathi and M. Atikuzzamman. 2018. Screening of lentil germplasm against stemphylium blight by observing disease reaction in three different stages. Malays. J. Halal. Res. 1(2): 15-18.

Abe, M., T. Yamada and H. Ohno. 2013. Dissolution of wet wood biomass without heating. RSC Adv. 4(33): 17136-17140.

Ahn, S. Y., S. Y. Eom, Y. H. Rhie, Y. M. Sung, C. E. Moon, G. M. Choi and D. J. Kim. 2013. Utilization of wood biomass char in a direct carbon fuel cell (DCFC) system. Appl. Energy. 105(1): 207-216.

Álvarez, C., F. M. Reyes-Sosa and B. Díez. 2016. Enzymatic hydrolysis of biomass from wood. Microb. Biotechnol. 9(2): 149-156.

Atikuzzamman, M., M. A. Islam, M. Moniruzzaman and M. S. Islam. 2018. Vendors and consumers status and microbiological analysis of some common fast food items of different fast food restaurants in Barisal City. Malays. J. Halal Res. 1(1): 17-21.

Ayieko, J., L. Abuogi, B. Simchowitz, E. A. Bukusi, A. H. Smith and A. Reingold. 2014. Efficacy of isoniazid prophylactic therapy in prevention of tuberculosis in children: A meta- analysis. BMC Infect. Dis. 14(1): 91.

Bryś, A., J. Bryś, E. Ostrowska-Ligęza, A. Kaleta, K. Górnicki, S. Głowacki and P. Koczoń. 2016. Wood biomass characterization by DSC or FT-IR spectroscopy. J. Therm. Anal. Calorim. 126(1): 27-35.

Candelier, K., S. Dumarçay, A. Pétrissans, M. Pétrissans, P. Kamdem and P. Gérardin. 2013. Thermodesorption coupled to GC- MS to characterize volatiles formation kinetic during wood thermodegradation. J. Anal. Appl. Pyrolysis. 101(5): 96-102.

Chen, J. T., C. Y. Ni, J. W. Lou and W. X. Peng. 2017. Molecules and functions of rosewood: Diospyros celebica. Arab. J. Chem. 11:756-762.

Chen, T. A. 2003. Study on organic volatile compounds in wood 
drying. World For. Res. 16(5): 30-34.

Dael, M. V., S. V. Passel, L. Pelkmans, R. Guisson, P. Reumermann, N. M. Luzardo, N. Witters and J. Broeze. 2013. A technoeconomic evaluation of a biomass energy conversion park. Appl. Energy. 104(2): 611-622.

Devora, I. G. E., A. R. Lizarraga, G. A. F. Weihs and J. A. Sanchez. 2017. Comparison of discharge methods for spill of brines, from a desalination plant in Sonora, Mexico. Rev. Int. Contam. Ambie. 33(SI): 45-54.

Duca, D., J. Lorv, C. L. Patten, D. Rose and B. R. Glick. 2014. Indole-3-acetic acid in plant-microbe interactions. Antonie Van Leeuwenhoek. 106(1): 85-125.

Eseyin, A. E and P. H. Steele. 2015. Current trends in the production and applications of torrefied wood/biomass - A review. Bioresources. 10(4): 515-707.

Fache, M., B. Boutevin and S. Caillol. 2016. Vanillin production from lignin and its use as a renewable chemical. ACS Sustain. Chem. Eng. 4(1): 6379-6401.

Fahim, N. F and Z. S. Sathi. 2018. Assesment of hepatoprotective activity of roots and barks of Achyranthes aspera in carbon tetrachloride-induced hepatotoxicity in rats. Malays. J. Halal. Res. 1(2): 23-26.

Fratzl, P. 2018. Materials Science Wood made denser and stronger. Nature. 554(7691): 172.

Fu, $\mathrm{H}$ and X. Liu. 2017. A study on the impact of environmental education on individuals' behaviors concerning recycled water reuse. Eurasia J. Math. Sci. Tech. Educ. 13(10): 6715-6724.

Ge, S. B., H. P. Gu, J. J. Ma, H. Q. Yang, S. C. Jiang and W. X. Peng. 2017. Potential use of different kinds of carbon in production of decayed wood plastic composite. Arab. J. Chem. 11: 838-843.

Ge, S. B., L. S. Wang, J. J. Ma, S. C. Jiang and W. X. Peng. 2018. Biological analysis on extractives of bayberry fresh flesh by GC- MS. Saudi. J. Biol. Sci. 25(4): 816-818.

Ge, S. B., L. S. Wang, Z. L. Liu, S. C. Jiang, X. Yang, W. Yang, W. X. Peng and T. Cai. 2018. Properties of nonvolatile and antibacterial bioboard produced from bamboo macromolecules by hot pressing. Saudi. J. Biol. Sci. 25(3): 474-478.

Ge, S. B., X. M. Chen, D. L. Li, Z. L. Liu, H. Ouyang, W. X. Peng and Z. F. Zhang. 2017. Hemicellulose structural changes during steam pretreatment and biogradation of Lentinus edodes. Arab. J. Chem. 11: 771-781.

Ge, S. B., Z. L. Liu, R. Li, Y. Furuta and W. X. Peng. 2017. Desulphurization characteristics of bamboo charcoal from sulfur solution. Saudi. J. Biol. Sci. 24(1): 127-131.

Ge, S. B., Z. L. Liu, Y. Furuta and W. X. Peng. 2017. Adsorption characteristics of sulfur solution by acticarbon against drinkingwater toxicosis. Saudi. J. Biol. Sci. 24(6): 1355-1360.

Ge, S. B., Z. L. Liu, Y. Furuta and W. X. Peng. 2017. Characteristics of activated carbon remove sulfur particles against smog. Saudi. J. Biol. Sci. 24(6): 1370-1374.

Ghosh, R., N. Nadiminty, J. E. Fitzpatrick, W. L. Alworth, T. J. Slaga and A. P. Kumar. 2015. Eugenol causes melanoma growth suppression through inhibition of E2F1 transcriptional activity. J. Biol. Chem. 280(7): 5812-9.

Gnonlonfin, G. J. B., A. Sanni and L. Brimer. 2012. Review scopoletin - A coumarin phytoalexin with medicinal properties. Crit. Rev. Plant. Sci. 31(1): 47-56.

Gołos, P and A. Kaliszewski. 2015. Aspects of using wood biomass for energy production. For. Res. Pap. 76(1): 78-87

Guerrini, G., E. Landi, K. Peiffer and A. Fortunato, A. 2018. Dry grinding of gears for sustainable automotive transmission production. J. Clean. Prod. 176: 76-88.
Han, Y., Q. Xu, J. N. Hu, X. Y. Han, W. Li and L. C. Zhao. 2015. Maltol, a food flavoring agent, attenuates acute alcohol-induced oxidative damage in mice. Nutrients. 7(1): 682-696.

Hino, F., M. Okazaki and Y. Miura. 2014. Effects of kinetin on formation of scopoletin and scopolin in tobacco tissue cultures. J. Agric Chem. Soc. Jpn. 46(3): 609-614.

Hong, S., Y. lizuka, T. Lee, C. Y. Kim and G. J. Seong. 2014. Neuroprotective and neurite outgrowth effects of maltol on retinal ganglion cells under oxidative stress. Mol. Vis. 20: 1456-1462.

Inman, M and C. J. Moody. 2012. Indole synthesis - something old, something new. Chem. Sci. 4(1): 29-41.

Jamuna, S., K. Karthika, S. Paulsamy, K. Thenmozhi, S. Kathiravan and R. Venkatesh. 2015. Confertin and scopoletin from leaf and root extracts of Hypochaeris radicata have anti-inflammatory and antioxidant activities. Ind. Crops. Prod. 70: 221-23.

Janevski, J. N., B. V. Stojanoviä, M. S. Lakoviä, M. M. Stojiljkoviä and D. M. Mitroviä. 2016. Wood biomass in Serbia resources and possibilities of use. Energ. Sour. 11(8): 732-738.

Jiang, S. C., S. B. Ge and W. X. Peng. 2017. Molecules and functions of rosewood: Dalbergia Stevenson. Arab. J. Chem. 11: 782-792.

Jiang, S. C., S. B. Ge, X. Wu, Y. M. Yang, J. T. Chen and W. X Peng. 2017. Treating n-butane by activated carbon and metal oxides. Toxicol. Environ. Chem. 99(5-6): 753-759.

Jiang, S. C., Y. F. Yang, S. B. Ge, Z. F. Zhang and W. X. Peng. 2017. Preparation and properties of novel flame-retardant PBS woodplastic composites. Arabian J. Chem. 11: 844-857.

Kaur, B and D. Chakraborty. 2013. Biotechnological and molecular approaches for vanillin production: A review. Appl. Biochem. Biotechnol. 169(4): 1353-1372.

Kim, N. Y., E. J. Jeon, S. H. Jung, S. J. Ahn, M. A. Park and J. S. Seo. 2018. Gene expression profiling and expression analysis of freshwater shrimp (Neocaridina Denticulata Denticulata) using expressed sequence tags and short-term exposure to copper. J. Environ. Biol. 39(1): 51-57.

Kim, Y. B., S. H. Oh, D. E. Sok and M. R. Kim. 2013. Neuroprotective effect of maltol against oxidative stress in brain of mice challenged with kainic acid. Nutr. Neurosci. 7(1): 33-39.

Kong, X. J., X. W. Liu, L. I. Jian-Yong and Y. J. Yang. 2013. Advance in pharmacological research of eugenol. Hubei Agric. Sci. 2013: 32.

Kopetz, H. 2013. Renewable resources: Build a biomass energy market. Nature. 494 (7435): 29.

Lan, T. 2007. Study on the Release of Organic Volatile Compounds from Pinus massoniana Wood During Drying. Beijing Forestry University.

Lange, J. P., E. van der Heide, J. van Buijtenen and R. Price. 2012. Furfural - A promising platform for lignocellulosic biofuels. Chemsuschem. 5(1): 150.

Lee, H. I., K. W. Yun, K. I. Seo, M. J. Kim and M. K. Lee. 2014. Scopoletin prevents alcohol-induced hepatic lipid accumulation by modulating the AMPK-SREBP pathway in diet-induced obese mice. Metabolism. 63(4): 593-601

Li, D. L., L. S. Wang, W. X. Peng, S. B. Ge and Y. Furuta. 2017. Chemical structure of hemicellulosic polymers isolated from bamboo bio-composite during mold pressing. polym. Composite. 38(9): 2009-2015.

Li, D. L., S. B. Ge, W. X. Peng, Q. D. Wu and J. G. Wu. 2017. Chemical structure characteristics of wood/lignin composites during mold pressing. Polym. Composite. 38(5): 955-965.

Liu, L., X. X. Cheng, W. W. Zhao, Y. H. Wang, X. Dong, L. L. Chen, D. Q. Zhang and W. X. Peng. 2017. Systematic characterization of volatile organic components and pyrolyzates from Camellia oleifera seed cake for developing high value-added products. 
Arab. J. Chem. 11: 802-814.

Liu, W., J. Hua, J. Zhou, H. Zhang, H. Zhu, Y. Cheng and R. Gust. 2012. Synthesis and in vitro antitumor activity of novel scopoletin derivatives. Bioorganic. Med. Chem. Lett., 22(15): 5008-5012.

Liu, Z. L., D. Q. Zhang and W. X. Peng. 2018. A novel ANFIS- PSO network for forecasting oil flocculated asphaltene weight percentage at wide range of operation conditions. Petrol. Sci. Technol. 36(14): 1044-1050.

Liu, Z. L., W. X. Peng, Y. Zare, D. Hui and K. Y. Rhee. 2018. Predicting the electrical conductivity in polymer carbon nanotube nanocomposites based on the volume fractions and resistances of the nanoparticle, interphase, and tunneling regions in conductive networks. RSC Adv. 8: 19001- 19010.

Lourençon, T. V., F. A. Hansel, T. A. D. Silva, L. P. Ramos and G. I. B. D. Muniz. 2015. Hardwood and softwood kraft lignins fractionation by simple sequential acid precipitation. Sep. Purif. Technol. 154: 82-88.

Mahato, S., S. Bhuju and J. Shrestha. 2018. Effect of trichoderma viride as biofertilizer on growth and yield of wheat. Malays. J. Sustain. Agric. 2(2): 1-5.

Martini, X., M. A. Hughes, J. A. Smith and L. L Stelinski. 2015. Attraction of redbay ambrosia beetle, Xyleborus glabratus, To leaf volatiles of its host plants in North America. J. Chem. Ecol. 41(7): 613-621.

Marvi, M., A. A. Pourbabaee, H. A. Alikhani, A. Haidari and Z. Manafi. 2016. The diversity of sulfur-oxidizing bacterial populations at an Iranian copper mine and the surrounding agricultural soils. Appl. Ecol. Env. Res. 14(3): 509-533.

Mathi, P., G. K. Veeramachaneni, K. K. Raj, V. R. Talluri, V. R. Bokka and M. Botlagunta. 2016. In vitro and in silico characterization of angiogenic inhibitors from Sophora interrupta. J. Mol. Model. 22(10): 247.

Min, D., Z. L. Liu, D. J. Yan and W. X. Peng. 2018. Application of LSSVM algorithm for estimating higher heating value of biomass based on ultimate analysis. Energy Sour. 40(6): 709-715

Moraski, G. C., L. D. Markley, P. A. Hipskind, H. Boshoff, S. Cho and S. G Franzblau. 2015. Miller corresponding, M. J. Advent of imidazo [1,2-a] pyridine-3-carboxamides with potent multiand extended drug resistant antituberculosis activity. ACS Med. Chem. Lett. 2(6): 466-470.

Muhammad, N., Z. Man and B. K. M. Azmi. 2012. Ionic liquid - A future solvent for the enhanced uses of wood biomass. Eur. J. Wood. Wood Prod. 70(1-3): 125-133.

Nishiguchi. S and T. Tabata. 2016. Assessment of social, economic, and environmental aspects of woody biomass energy utilization: Direct burning and wood pellets. Renew. Sust. Energ. Rev. 57: 1279-1286.

Nosek, R., M. Holubcik and J. Jandacka. 2015. The impact of bark content of wood biomass on biofuel properties. Bioresources. 11(1): 44-53

Ouyang, H., K. Hou, L. Wang and W. X. Peng. 2016. Optimization protocol for the microwave-assisted extraction of antioxidant components from Pinus elliottii needles using response surface methodology. Bio. Res. 12(1): 478-494.

Patrik, B., M. Vladimír, M., D. Martin, D., Z. Pavel, Z. and R. Kazimierz. 2017. Review of energy potential of the wood biomass of orchards and vineyards in the Czech Republic. Res. Agric. Eng. 63(SI): S1-S7.

Peng, W. X, A. Maleki, M. A. Rosen and P. Azarikhah. 2018. Optimization of a hybrid system for solar-wind-based water desalination by reverse osmosis: Comparison of approaches. Desalination. 442: 16- 31.
Peng, W. X., D. L. Li, M. L. Zhang, S. B. Ge, B. Mo, S. S. Li and M. Ohkoshi. 2017. Characteristics of antibacterial molecular activities in poplar wood extractives. Saudi. J. Biol. Sci. 24(2): 399-404.

Peng, W. X., L. S. Wang, M. Mirzaee, H. Ahmadi, M. J. Esfahani and S. Fremaux. 2017. Hydrogen and syngas production by catalytic biomass gasification. Energy Convers. Manag. 135: 270-273.

Peng, W. X., S. B. Ge, Z. L. Liu and Y. Furuta., 2017. Adsorption characteristics of sulfur powder by bamboo charcoal to restrain sulfur allergies. Saudi. J. Biol. Sci. 24(1): 103-107.

Peng, W. X., Z. Lin, L. S. Wang, J. B. Chang, F. L. Gu and X. W. Zhu. 2016. Molecular characteristics of Illicium verum extractives to activate acquired immune response. Saudi. J. Biol. Sci. 23(3): 348-352.

Peng, W. X., Z. L. Liu, M. Motahari-Nezhad, M. Banisaeed, S. Shahraki and M. Beheshti. 2016. A detailed study of oxy-fuel combustion of biomass in a circulating fluidized bed (CFB) combustor: Evaluation of catalytic performance of metal nanoparticles (Al, Ni) for combustion efficiency improvement. Energy. 109: 1139-1147.

Poesen, R., L. Viaene, K. Verbeke, P. Augustijns, B. Bammens, K. Claes, D. Kuypers, P. Evenepoel and B. Meijers. 2014. Cardiovascular disease relates to intestinal uptake of $p$-cresol in patients with chronic kidney disease. BMC Nephrol. 15(1): 87.

Roubach, R., L. C. Gomes, F. A. Leão Fonseca and A. L. Val. 2015. Eugenol as an efficacious anaesthetic for tambaqui, Colossoma macropomum (Cuvier). Aquacult. Res., 36(11): 1056-1061.

Roy, M. M and K. W. Corscadden. 2012. An experimental study of combustion and emissions of biomass briquettes in a domestic wood stove. Appl. Energ. 99(2): 206-212.

Seefeld, M. A., W. H. Miller, K. A. Newlander, W. J. Burgess, W. E. DeWolf and P. A. Elkins. 2015. Indole naphthyridinones as inhibitors of bacterial enoyl-ACP reductases Fabl and FabK. J. Med. Chem. 46(9): 1627-1635.

Seo, E. J., M. Saeed, B. Y. Law, A. G. Wu, O. Kadioglu, H. J. Greten and T. Efferth. 2016. Pharmacogenomics of scopoletin in tumor cells. Molecules. 21(4): 496.

Shabani, N. and T. Sowlati. 2013. A mixed integer non-linear programming model for tactical value chain optimization of a wood biomass power plant. Appl. Energ. 104(4): 353-361.

Sillett, S. C., R. V. Pelt, G. W. Koch, A. R. Ambrose, A. L. Carroll, M. E. Antoine and B. M. Mifsud. 2015. Increasing wood production through old age in tall trees. For. Ecol. Manag. 259(5): 976-994.

Song, J. W., C. J. Chen, S. Z. Zhu, M. W. Zhu, J. Q. Dai, U. Ray, Y. J. Li, Y. D. Kuang, Y. F. Li and N. Quispe. 2018. Processing bulk natural wood into a high-performance structural material. Nature. 554(7691): 224.

Sun, C. Y., H. H. Hsu and M. S. Wu. 2013. P-cresol sulfate and indoxyl sulfate induce similar cellular inflammatory gene expressions in cultured proximal renal tubular cells. Nephrol. Dial. Trans. 28(1): 70-8.

Sun, Q. X., Z. H. Peng and Q. S. Zhang. 2004. Volatile composition of Chinese fir wood under natural condition and its effects on human physical and mental health. J. Anhui. Agric. Coll. 31(2): 158-163.

Tabana, Y. M., L. E. Hassan, M. B. Ahamed, S. S. Iqbal, M. A. Saeed, M. S. Khan, D. Sandai, A. S. Majid, C. E. Oon and A. M. Majid. 2016. Scopoletin, an active principle of tree tobacco (Nicotiana glauca) inhibits human tumor vascularization in xenograft models and modulates ERK1, VEGF-A, and FGF-2 in computer model. Microvasc. Res. 107: 17-33.

Tollefson, J. 2017. Wood grows up. Nature. 545: 7654. 
Wang, L. S., S. B. Ge, Z. L. Liu, Y. F. Zhou, X. X. Yang, W. Yang, D. L. Li and W. X. Peng. 2018. Properties of antibacterial bioboard from bamboo macromolecule by hot press. Saudi. J. Biol. Sci. 25(3): 465-468.

Weldu, Y. W., G. Assefa and O. Jolliet. 2017. Life cycle human health and ecotoxicological impacts assessment of electricity production from wood biomass compared to coal fuel. Appl. Energ. 187: 564-574.

Wu, Q., S. Zhang, B. Hou, H. Zheng, W. Deng, D. Liu and W. Tang. 2015. Study on the preparation of wood vinegar from biomass residues by carbonization process. Bioresour. Technol. 179: 98-103.

Wu, Y. Q., X. G. Li, Y. F. Zuo, X. J. Li, Y. Qing and X. L. Zhang. 2016. Research status on the utilization of forest and agricultural biomass in inorganic wood-based panel. J. For. Eng. 11:902-993.

Xie, Y. Z., S. B. Ge, S. C. Jiang, Z. L. Liu, L. Chen, L. S. Wang, J. T. Chen, L. C. Qin and W. X. Peng. 2018. Study on biomolecules in extractives of Camellia oleifera fruit shell by GC-MS. Saudi J Biol Sci. 25(2): 234-236.

Xu, K. Q., G. X. He, J. M. Qin, X. X. Cheng, H. J. He, G. X. He, J. M. Qin, X. X. Cheng, H. J. He, D. Q. Zhang and W. X. Peng.
2018. High-efficient extraction of principal medicinal components from fresh Phellodendron bark (cortex phellodendri). Saudi. J. Biol. Sci. 25(4): 811-815.

Yang, A., Y. Han, S. Li, H. Xing, Y. Pan and W. Liu. 2017. Synthesis and comparison of photocatalytic properties for bi2wo6 nanofibers and hierarchical microspheres. J. Alloy. Compd. 695: 915-921.

Yao, X., Z. Ding, Y. Xia, Z. Wei, Y. Luo, C. Feleder and Y. Dai. 2012. Inhibition of monosodium urate crystal-induced inflammation by scopoletin and underlying mechanisms. Int. Immunopharmacol. 14(4): 454-62.

Zhao, P., L. Chen, L. H. Li, Z. F. Wei, B. Tong, Y. G. Jia, L. Y. Kong, Y. F. Xia and Y. Dai. 2014. SC-III3, a novel scopoletin derivative, induces cytotoxicity in hepatocellular cancer cells through oxidative DNA damage and ataxia telangiectasia-mutated nuclear protein kinase activation. BMC Cancer. 14(1): 987.

Zhao, Y. 2012. Auxin biosynthesis: A simple two-step pathway converts tryptophan to indole-3-acetic acid in plants. Section of cell. Dev. Biol. 5(2): 334-8.

Zhou, J., W. Zhao, K. Tang and W. Peng. 2016. Seismic performance of square, thin-walled steel tube/bamboo plywood composite hollow columns with binding bars. Soil. Dyn. Earthq. Eng. 89: 152-162. 